\title{
Clinical and radiographic comparison of implants in regenerated or native bone: 5-year results
}

\author{
Benić, G I ; Jung, R E ; Siegenthaler, D W ; Hämmerle, C H F
}

\begin{abstract}
PURPOSE: The aim of this study was to test whether or not implants associated with bone regeneration show the same survival and success rates as implants placed in native bone in patients requiring both forms of therapy. MATERIAL AND METHODS: Thirty-four patients (median age of 60.3 years, range 18-77.7 years) had been treated 5 years before the follow-up examination. Machined screw-type implants were inserted following one of two surgical procedures: (1) simultaneously with a guided bone regeneration (GBR) procedure, which involved grafting with xenogenic bone substitute material, autogenous bone or a mixture of the two and defect covering with a bio-absorbable collagen membrane (test) and (2) standard implantation procedure without bone regeneration (control). For data recording, one test and one control implant from each patient were assessed. Examination included measurements of plaque control record (PCR), probing pocket depth (PPD), bleeding on probing (BOP), width of keratinized mucosa (KM), frequency of situations with supra-mucosal location of the crown margin, implant survival assessment and radiographic examination. Radiographs were digitized to assess the marginal bone level (MBL). Differences between groups were tested using the one-sample t-test. The estimation of survival rate was based on Kaplan-Meier analysis. RESULTS: The follow-up period of the 34 GBR and 34 control implants ranged from 49 to 70 months (median time 57 months). Cumulative survival rates reached $100 \%$ for the GBR group and $94.1 \%$ for the control group without statistical significance. No statistically significant differences for clinical and radiographic parameters were found between the two groups regarding PCR, BOP, PPD, KM and MBL. CONCLUSION: The present study showed that, clinically, implants placed with concomitant bone regeneration did not performed differently from implants placed into native bone with respect to implant survival, marginal bone height and peri-implant soft tissue parameters.
\end{abstract}

DOI: https://doi.org/10.1111/j.1600-0501.2008.01583.x

Posted at the Zurich Open Repository and Archive, University of Zurich

ZORA URL: https://doi.org/10.5167/uzh-19630

Journal Article

Accepted Version

Originally published at:

Benić, G I; Jung, R E; Siegenthaler, D W; Hämmerle, C H F (2009). Clinical and radiographic comparison of implants in regenerated or native bone: 5-year results. Clinical Oral Implants Research, 20(5):507-513.

DOI: https://doi.org/10.1111/j.1600-0501.2008.01583.x 


\title{
Clinical and radiographic comparison of implants in regenerated or native bone: 5-year results
}

\section{Goran I. Benić, Ronald E. Jung, David W. Siegenthaler, Christoph H.F. Hämmerle}

\author{
University of Zurich, Clinic for Fixed and Removable Prosthodontics and Dental \\ Material Science, Switzerland
}

Key words: dental implants; bone regeneration; human; membranes; split mouth design; long-term

Running title: implants in regenerated or native bone

Address for correspondence:

Prof. Dr. Ch. Hämmerle

Clinic for Fixed and Removable Prosthodontics and

Dental Material Science

Center for Dental and Oral Medicine and

Cranio-Maxillofacial Surgery

University of Zurich

Plattenstrasse 11

8032 Zürich

Switzerland

WebSite: www.uzh.ch

E-mail: christoph.hammerle@zzmk.uzh.ch 


\section{Abstract}

Purpose: The aim of this study was to test whether or not implants associated with bone regeneration show the same survival and success rates as implants placed in native bone in patients requiring both forms of therapy

Material and Methods: 34 patients (median age of 60.3 years, range 18 to 77.7 years) had been treated 5 years prior to the follow-up examination. Machined screwtype implants were inserted following one of two surgical procedures: 1. simultaneously with a GBR procedure, which involved grafting with xenogenic bone substitute material, autogenous bone or a mixture of the two and defect covering with a bio-absorbable collagen membrane (test); 2 . standard implantation procedure without bone regeneration (control). For data recording one test and one control implant from each patient were assessed. Examination included measurements of plaque control record (PCR), probing pocket depth (PPD), bleeding on probing (BOP), width of keratinized mucosa $(\mathrm{KM})$, frequency of situations with supra-mucosal location of the crown margin, implant survival assessement and radiographic examination.

Radiographs were digitized to assess the marginal bone level (MBL). Differences between groups were tested using the one sample $t$ test. The estimation of survival rate was based on Kaplan-Meier analysis.

Results: The follow-up period of the 34 GBR and 34 control implants ranged from 49 to 70 months (median time 57.0 months). Cumulative survival rates reached $100 \%$ for the GBR group and $94.1 \%$ for the control group without statistical significance. No statistically significant differences for clinical and radiographic parameters were found between the 2 groups regarding PCR, BOP, PPD, KM and MBL. 
Conclusion: The present study showed that implants placed with concomitant bone regeneration performed clinically not different than implants placed into native bone with respect to implant survival, marginal bone height and peri-implant soft tissue parameters. 


\section{Introduction}

The requirement for successful osseointegration of dental implants is a sufficient quantity and quality of osseous tissue to ensure the stabilization of the implant and to allow for bone-to-implant contact of the entire surface intended for osseointegration (Albrektsson et al. 1981; Adell 1985). In cases of insufficient quantity of bone tissue at the site of implantation decreased implant success rates have been reported (Lekholm et al. 1986).

Based on these clinical findings different techniques have been developed to increase the bone volume. These techniques encompass:

Osteoinduction (Urist 1965; Reddi et al. 1987), distraction osteogenesis (Ilizarov 1989a, 1989b), osteoconduction (Burchardt 1983; Reddi et al. 1987), and guided bone regeneration (GBR) (Dahlin et al. 1988, 1989, 1991a; Kostopoulos \& Karring 1994; Nyman \& Lang 1994; Hämmerle et al. 1996).

The GBR technique is highly successful for the treatment of peri-implant bone defects (Becker et al. 1991; Dahlin et al. 1995; Brägger et al. 1997; Hämmerle et al. 1997, 1998; Simion et al. 2001) and for bone regeneration prior to implant placement in localized ridge defects (Lang et al. 1994; Buser et al. 1990; Nyman et al. 1990; Hämmerle et al. 2007). Thus the application of GBR provides the clinicians with the ability to place implants in areas of insufficient amounts of bone.

An important question is whether or not implants placed in sites associated with bone regeneration provide survival and success rates similar to the ones of implants placed in sites with sufficient native bone. Two recent systematic reviews studied the survival of implants in bone sites regenerated with GBR in partially edentulous patients (Hämmerle et al. 2002; Fiorellini et al. 2003). In one review 11 studies providing at least 12 months results following prosthetic reconstruction could be identified (Hämmerle et al. 2002). Only 2 of them had both test and control implants included in their analysis (Mayfield et al. 1998; Zitzmann et al. 2001). The methodological quality 
of the other reported studies was generally not very high. Although, based on these scarce data it was concluded that survival and success rates were not significantly different between implants in regenerated and non-regenerated bone. A more recent systematic review (Fiorellini et al. 2003) included 13 studies identified another one with a test and control group (Corrente et al. 2000). The results reported in this systematic review confirmed the ones from the first one.

The highest evidence can be gathered from clinical studies including implants in regenerated and non-regenerated bone in patients requiring both forms of therapy.

The aim of the present study was to test whether or not implants associated with bone regeneration show the same survival and success rates as implants placed in native bone in patients requiring both forms of therapy. 


\section{Material and Methods}

This cross-sectional retrospective study included all patients of the Departement of Fixed and Removable Prosthodontics and Dental Material Science, University of Zurich, Switzerland, who had received implants both in sites with regenerated and with non-regenerated bone 5 years prior to the follow-up examination. The 41 patients included in this study were invited by sending them a single letter to attend the examination. Four letters were returned because the patients had moved away and 3 patients declined to attend, rendering a recall rate of $83 \%$.

A total of 34 patients participated in this study presenting with a median age of 60.3 years (range 18-77.7 years). 19 patients were women and 15 were men.

\section{Clinical procedures}

Depending on the quantity of the bone tissue at the implantation site implants were inserted following one of the two specified surgical procedures:

- simultaneously with GBR for the treatment of osseous defects including dehiscences, fenestrations and infrabony defects. The GBR procedure involved grafting with a xenogenic bone substitute mineral (Bio-Oss ${ }^{\circledR}$ spongiosa granules, Geistlich AG, Wolhusen, Switzerland), autogenous bone primarily harvested from the site of surgery or a mixture of the two. The site was covered with a bio-resorbable collagen membrane (Bio-Gide ${ }^{\circledR}$, Geistlich AG, Wolhusen, Switzerland).

- standard implantation procedure executed in situations with bone volumes sufficient for complete coverage of the endosseous implant surface.

All implants exhibited a machined endosseous surface (Brånemark System, Nobel Biocare, Gothenburg, Sweden).

The implant placement was never performed as a type I procedure but rather as type II and III or type IV procedures (Hämmerle et al. 2004). 
The sites of implantation were primarily closed for a submerged healing. The postoperative protocol included rinsing with a $0.2 \%$ chlorhexidine solution twice daily, $375 \mathrm{mg}$ of penicillin tid for 5 days, and a non-steroidal anti-inflammatory medication according to individual requirements.

The implants were loaded after a minimum healing time of 6 months. There was one exception where healing was obtained with the implant in transmucosal position and prosthetic loading 3 weeks after implantation.

A total of 34 patients were available for the follow up examination and could be inserted in the study. They received 68 implants. 35 implants were placed in the mandible and 33 in the maxilla. In the control group (standard implant placement) there were 18 mandibular and 16 maxillary implants, and in the GBR group 17 mandibular and 17 maxillary implants.

47 implants presented with a regular platform (RP), 19 a wide platform (WP) and 2 a narrow platform (NP). The distribution was similar in both treatment groups. There were $23 \mathrm{RP}, 10 \mathrm{WP}$ and $1 \mathrm{NP}$ implants in the control and $24 \mathrm{RP}, 9 \mathrm{WP}$ and $1 \mathrm{NP}$ implants in the GBR group.

Considering implant length the distribution between the groups was similar as well (Table 1).

\section{Follow-up examination}

Prior to clinical and radiographic examination, a thorough medical history was obtained from each patient. All patients were examined by one examiner.

For data recording one test and one control implant from each patient were assessed. In situations where two or more implants in the test and/or control group were present in a particular patient, implants to be analyzed were chosen following these hierarchical criteria:

1. test and control implants located in the same region (anterior, posterior) 
2. test and control implants located in the same jaw

3. in all other situations test and control implants were randomly chosen by casting a die

The following clinical parameters were assessed:

- plaque control record (PCR) (O'Leary 1972)

- probing pocket depth to the nearest millimeter at six sites around the implant (PPD) (Ramfjord 1959)

- bleeding on probing at six sites around the implant (BOP) (Ainamo \& Bay 1975)

- width of the keratinized mucosa at the mid-buccal aspect of the implants (KM)

- frequency of situations with supra-mucosal location of the crown margin at the mid buccal aspect, in addition

- implant survival was recorded.

\section{Radiographic analysis}

For the evaluation of the marginal bone level intraoral radiographs were taken using the long-cone paralleling technique with the central beam aiming at the alveolar crest (Updegrave 1968).

The images were digitized and the marginal bone level (MBL) (distance between the top of implant shoulder and the first visible bone-to-implant contact) was measured on the mesial and distal aspect of every implant using a $10-15 \mathrm{x}$ magnification (Buser 1991; Weber 1992). The measured distance between three implant threads was used as the basis for the calibration and determination of the exact magnification and distortion of the images (Rodoni et al. 2005) (Figure 1).

All measurements were performed by two examiners. In cases of disagreement the values were discussed until an agreement was found. 
Figure 1: Assessment of the distance from the shoulder to the first bone-to-implant contact on digitized radiographs (original magnification $\times 12.5$ )

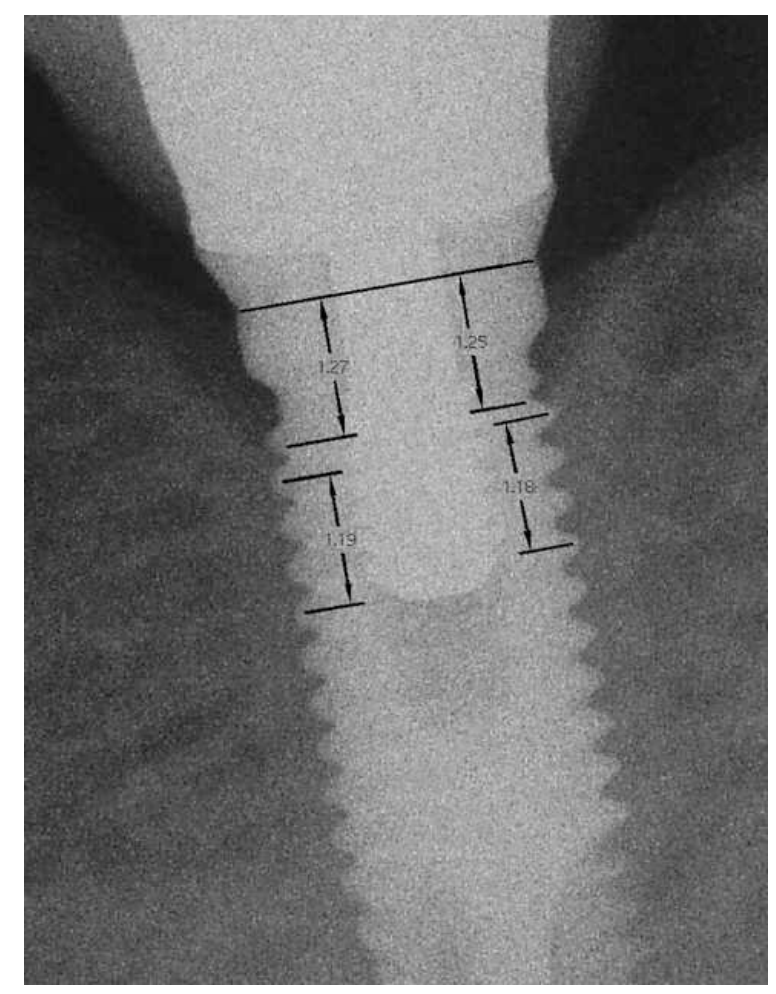

\section{Statistical analysis}

A statistical software program Stat View 5.0.1 was used for all statistical analyses.

The estimation of the implant survival rate was based on Kaplan-Meier analysis and group comparison was made using the log rank test.

For the statistical analysis the six values around each implant for PCR, PPD, BOP and the two values of the radiographic assessment of the bone level mesially and distally were averaged to one value each. Only the cases with both implants in native and regenerated bone were used for the analysis.

The data distribution was plotted with boxplots and characterized by mean values and standard deviations. In order to detect differences between the test and the control group one sample $t$ test together with the corresponding $95 \%$ confidence 
interval $(\mathrm{CI})$ were applied to differences of mean PCR, mean PPD, mean BOP, mean MBL and KM values. Results of the tests with p-values $<5 \%$ were considered significant.

The power of the one sample $t$ test was computed setting relevant differences at $20 \%$ for PCR, $20 \%$ for BOP, $2 \mathrm{~mm}$ for PPD, $2 \mathrm{~mm}$ for KM and $1 \mathrm{~mm}$ for MBL.

In order to control for multiple testing Benjamini and Hochberg False Discovery Rate was applied.

Correlation analysis and subsequent unpaired $t$ test were performed to investigate the influence of various parameters (age, smoking, parafunctional acivities, history of periodontal disease) on the main endpoints. 


\section{Results}

The follow-up period after implant placement ranged from 49 to 70 months (median time 57.0 months).

Patient history revealed that 8 individuals smoked more than 10 cigarettes a day. Two patients had a diagnosis of type 2 diabetes. Out of 3 patients with a history of malignant tumors, two reported chemo- and radiotherapy in the past 5 years, i.e. after placement of the implants analyzed in this study.

6 patients reported parafunctional activities during the day or the night.

The review of the patients' periodontal charts before the implant placement showed that 12 patients (35\%) had periodontal disease. They underwent periodontal therapy and the implants were only placed following successful treatment of the periodontal disease.

The cumulative implant survival rate at the 5-year examination reached $100 \%$ for the GBR group. In the control group two implants were lost and the cumulative survival rate was $94.1 \%$. The difference was not statistically significant. One control implant was lost 5 months after implant placement. This implant had been loaded only 3 weeks after implantation and subsequently became mobile. In another patient one control implant was not osseointegrated as detected at the time of the abutment connection, 6 months after implantation.

In all patients except the one mentioned above the healing time until loading was equal for the test and control implants and prosthesis incorporation was performed 9 to 10 months after implantation.

The majority of the implants were reconstructed with fixed prostheses: 13 with single-tooth and 43 with splinted reconstructions. The other 11 implants supported removable prostheses: 3 with ball attachments and 8 with bars. The proportions of different types of restorations were similar in both treatment groups (Table 2). 
The results of the clinical measurements of plaque control record, bleeding on probing, probing pocket depth, width of the keratinized mucosa and the radiographic height of the marginal bone are presented in Tables 3,4 and the differences between two groups in the Table 5.

The two cases with lost implants were omitted from the statistical analysis of the following clinical and radiographic parameters.

Overall plaque scores within the entire dentition amounted to $25.8 \% \pm 12.8 \%$ (SD), ranging from $5 \%$ to $56 \%$ in the 32 patients. Full mouth plaque score was significantly higher in men $(34 \% \pm 13 \%)$ compared to women $(20 \% \pm 10 \%)(\mathrm{p} \leq 0.05)$.

The mean plaque control record at the implant sites reached $26 \% \pm 16 \%$ (SD) for control implants and $28 \% \pm 21 \%$ for GBR-treated sites without a significant difference between the two groups (Table $3,4,5$ ).

Mean BOP values were similar for both groups $\left(\mathrm{BOP}_{\text {control }} 47 \% \pm 26 \%\right.$ (SD), $\left.\mathrm{BOP}_{\mathrm{GBR}} 51 \% \pm 23 \%\right)($ Table 3, 4, 5).

In addition, no significant difference between the two groups was found regarding mean probing pocket depth $\left(\mathrm{PPD}_{\text {control }} 3.1 \mathrm{~mm} \pm 0.6 \mathrm{~mm}(\mathrm{SD}), \mathrm{PPD}_{\mathrm{GBR}}\right.$ $3.2 \mathrm{~mm} \pm 0.6 \mathrm{~mm})($ Table $3,4,5)$.

The keratinized mucosa at the buccal aspect was present in the majority of sites: 94\% for the control and 97\% for the GBR group. When measuring the height of the keratinized mucosa at the buccal aspect of the implants, similar mean values were found in the groups (control group $2.3 \pm 1.2 \mathrm{~mm}(\mathrm{SD})$, GBR group $2.8 \pm 1.4 \mathrm{~mm}$ ). No statistically significant differences were noted (Table 3, 4, 5).

With respect to the location of the crown-abutment junctions, $16 \%$ of the implants in the control group and $12 \%$ in the GBR group exhibited a supra-mucosal margin at the mid buccal aspect.

Radiographic evaluation indicated that all implants were successfully osseointegrated as seen by close bone to implant contact from the alveolar crest to the 
apical end of the implant. The radiographically determined mean marginal bone level (MBL) amounted to $1.6 \pm 0.9 \mathrm{~mm}(\mathrm{SD})$ for the control group and $1.3 \pm 0.5 \mathrm{~mm}$ for the GBR group. The difference of $0.3 \pm 0.6 \mathrm{~mm}$ between the control and the test group was not statistically significant (Table 3, 4, 5).

The power of the one sample $t$ test was computed for the present sample size. It showed more than $90 \%$ power for BOP and more than 95\% for PCR, PPD, KM, and MBL.

The patients' age range was quite large, but it had no impact on the mean endpoints. Neither did smoking habits, parafunctional activities, and history of periodontal disease have an influence on the clinical and radiographic results. 


\section{Discussion}

The results of the present study demonstrated implants placed into sites with bone regeneration to be as successful as implant placed into sites with native bone. No difference between the survival rates were found for the two groups.

Although, the cumulative survival rate was lower for the implants placed into native bone $(94.1 \%, 100 \%$ for the GBR group) this difference was not statistically significant. These results compare well with systematic reviews reporting survival rates of implants placed into sites with regenerated bone ranging from $79 \%$ to $100 \%$, with the majority of studies indicating more than $90 \%$ after at least 1 year of function (Hämmerle et al. 2002; Fiorellini et al. 2003).

The survival rates obtained in these systematic reviews were similar to those reported in the meta-analysis of prospective longitudinal studies for implant therapy with and without bone regeneration after at least 5 years (Berglundh et al. 2002).

Implants were placed according to the manufacturer's instructions thus placing the implant shoulder at the level of the bone crest. In situations with bone defects GBR was performed in order to cover the exposed surface and to regenerate the bone to the level of the implant shoulder similar to the situation of implants placed into native bone. In this retrospective study the bone level at follow up and not the change in crestal bone level were measured. Nevertheless, since the level of the marginal bone is supposed to be initially similar in both groups, measurements of absolute bone levels at the follow-up examination are expected to reflect changes over time.

The level of the marginal bone was 1.3 to $1.6 \mathrm{~mm}$ below the shoulder of the implant at the 5-year follow-up examination. These results demonstrated that bone regenerated by GBR in peri-implant bone defects remains as stable over time as pristine peri-implant bone.

These data compare well with results reported in previous studies including implants in pristine as well as regenerated bone (Zitzmann et al. 2001; Mayfield et al. 
1998). Values of 1.73 for the control and $1.83 \mathrm{~mm}$ for the test group regarding marginal bone level were reported in one study 5 years after implant placement (Zitzmann et al. 2001). Although, this difference was small statistical analysis revealed it to be significant. Bone regeneration had been performed using bioresorbable collagen membranes supported by a deproteinized bovine bone mineral.

In another controlled study of shorter duration a mean radiographic bone loss of $0.6 \mathrm{~mm}$ for control and $0.7 \mathrm{~mm}$ for test implants was observed 23-27 months following incorporation of the fixed reconstructions (Mayfield et al. 1998). This difference was statistically non-significant. Local bone dehiscences and fenestrations defects had been treated using a bioresorbable PLA-PGA membrane without a supporting material.

The level of the marginal bone was investigated in a 5-year study involving 12 sites primary regenerated with ePTFE membranes and autogenic bone without a control group (Buser et al. 1996). A bone level change of $0.8-1.3 \mathrm{~mm}$ was reported between implant placement and 5 years, which is slightly less than in the present study. In this particular study, however a one-piece implant was used and not a two-piece as in the present study. In addition, the staged approach was chosen, where the bone is first regenerated and the implant is subsequently placed into a ridge exhibiting sufficient bone volume.

The marginal bone level values for the control and GBR group in the present study are in accordance with the ones observed in long-term studies documenting the outcome of implants placed in native bone under standard conditions (Adell et al. 1981; Pylant et al. 1992; Jemt \& Lekholm 1993; Lekholm et al. 1994; Weber et al. 2000).

In summary, the marginal bone levels in the present controlled study were within the range of previously reported values. It should be noted, however, that those studies were frequently of shorter duration and only very few of them included a control group. 
In the present study the analysis of the clinical parameters revealed no differences between two groups regarding prevalence of plaque, bleeding on probing, probing pocket depth, the height of the keratinized mucosa and frequency of supramucosally located crown margins.

\section{Conclusion}

Based on the results of this 5-year cross-sectional controlled study it is concluded that implants placed in regenerated bone exhibit a clinical performance similar to implants placed into native bone with respect to implant survival, marginal bone height and peri-implant soft tissue parameters. The prevalence of plaque present in the dentition and in the study implants as well as the BOP values were quite high in this study. Nevertheless, the overall survival rate of the implants in the control and in the test group was high and the marginal bone levels within the range of previously published data.

\section{Acknowledgements}

The investigators would like to gratefully acknowledge the statistical assistance of the Department of Biostatistics, University of Zurich. The clinical support of the faculty of the Clinic for Fixed and Removable Prosthodontics and Dental Material Science of the University of Zurich are highly appreciated. 
Table 1: Distribution of implant lengths presented as absolute and relative numbers

\section{Group}

$\begin{array}{cccc}\text { Implant Length (mm) } & \text { GBR }(\mathbf{n = 3 4}) & \text { Control }(\mathbf{n = 3 4}) & \text { Total }(\mathbf{n = 6 8}) \\ 7 & 1(2.9 \%) & 1(2.9 \%) & 2(2.9 \%) \\ 8.5 & 6(17.6 \%) & 6(17.6 \%) & 12(17.6 \%) \\ 10 & 9(26.5 \%) & 10(29.4 \%) & 19(28.0 \%) \\ 11.5 & 9(26.5 \%) & 7(20.6 \%) & 16(23.5 \%) \\ 13 & 7(20.6 \%) & 7(20.6 \%) & 14(20.6 \%) \\ 15 & 2(5.9 \%) & 3(8.9 \%) & 5(7.4 \%)\end{array}$


Table 2: Distribution of different types of restorations presented as absolute and relative numbers

\begin{tabular}{lccc} 
& \multicolumn{2}{c}{ Group } \\
\cline { 2 - 3 } Restoration type & GBR $(\mathbf{n = 3 4 )}$ & Control $(\mathbf{n = 3 3 )}$ & Total $(\mathbf{n = 6 7 )}$ \\
Fixed single & $6(17.7 \%)$ & $7(21.2 \%)$ & $13(19.4 \%)$ \\
Fixed connected & $22(64.7 \%)$ & $21(63.6 \%)$ & $43(64.2 \%)$ \\
Removable single & $1(2.9 \%)$ & $2(6.1 \%)$ & $3(4.5 \%)$ \\
Removable connected & $5(14.7 \%)$ & $3(9.1 \%)$ & $8(11.9 \%)$
\end{tabular}


Table 3: Results of the clinical and radiographic measurements for the $\mathbf{3 2}$ control group implants

$\begin{array}{lccccc} & \text { PCR (\%) } & \text { BOP (\%) } & \text { PPD (mm) } & \text { KM (mm) } & \text { MBL (mm) } \\ \text { mean } & 26 & 47 & 3.1 & 2.3 & 1.6 \\ \text { standard deviation } & 16 & 26 & 0.6 & 1.2 & 0.86 \\ \text { median } & 33 & 50 & 3.0 & 2.0 & 1.62 \\ \text { range } & 0-67 & 0-100 & 2.2-4.7 & 0-5 & 0.07-3.64\end{array}$

$\mathrm{PCR}=$ Plaque control record $; \mathrm{BOP}=$ Bleeding on probing; $\mathrm{PPD}=$ probing pocket depth; $\mathrm{KM}=$ width of keratinized mucosa; $\mathrm{MBL}=$ marginal bone level 
Table 4: Results of the clinical and radiographic measurements for the 32 GBR group implants

$\begin{array}{lccccc} & \text { PCR (\%) } & \text { BOP (\%) } & \text { PPD (mm) } & \text { KM (mm) } & \text { MBL (mm) } \\ \text { mean } & 28 & 51 & 3.2 & 2.8 & 1.33 \\ \text { standard deviation } & 21 & 23 & 0.6 & 1.4 & 0.51 \\ \text { median } & 17 & 50 & 3.3 & 3.0 & 1.34 \\ \text { range } & 0-67 & 17-100 & 2.3-4.3 & 0-6 & 0-2.36\end{array}$

$\mathrm{PCR}=$ Plaque control record $; \mathrm{BOP}=$ Bleeding on probing; $\mathrm{PPD}=$ probing pocket depth; $\mathrm{KM}=$ width of keratinized mucosa; $\mathrm{MBL}=$ marginal bone level 
Table 5: Differences of mean results between GBR and control group

\begin{tabular}{|c|c|c|c|c|c|c|c|}
\hline & & & & nge & & & \\
\hline & mean & SD & minimun & maximum & $\begin{array}{l}95 \% \\
\text { lower }\end{array}$ & $\begin{array}{l}95 \% \\
\text { upper }\end{array}$ & $\begin{array}{c}\text { statistical } \\
\text { significance }\end{array}$ \\
\hline $\mathrm{PCR}_{\mathrm{GBR}}-\mathrm{PCR}_{\text {control }}(\%)$ & 2 & 21 & -34 & 34 & -5 & 10 & 0.57 \\
\hline BOP $_{\mathrm{GBR}}-\mathrm{BOP}_{\text {control }}(\%)$ & 4 & 31 & -66 & 50 & -7 & 15 & 0.57 \\
\hline $\mathrm{PPD}_{\mathrm{GBR}}-\mathrm{PPD}_{\text {control }}(\mathrm{mm})$ & 0.1 & 0.5 & -1 & 1.3 & -0.08 & 0.29 & 0.44 \\
\hline$K M_{G B R}-K M_{\text {control }}(\mathrm{mm})$ & 0.5 & 1.6 & -2 & 6 & -0.04 & 1.1 & 0.17 \\
\hline$M B L_{G B R}-M B L_{\text {control }}(\mathrm{mm})$ & -0.3 & 0.6 & -1.3 & 1 & -0.49 & -0.05 & 0.08 \\
\hline
\end{tabular}

$\mathrm{PCR}=$ Plaque control record $; \mathrm{BOP}=$ Bleeding on probing; $\mathrm{PPD}=$ probing pocket depth; $\mathrm{KM}=$ width of keratinized mucosa; $\mathrm{MBL}=$ marginal bone level; $\mathrm{SD}=$ standard deviation; $\mathrm{CI}=$ confidence interval 


\section{References}

Adell, R. (1985) Long-term treatment results. In: Brånemark, P.-I., Zarb, G.A., Albrektsson, T., eds. Tissue-Integrated Prostheses - Osseointegration in Clinical Dentistry, p. 175-186. Chicago: Quintessence

Adell, R., Lekholm, U., Rockeler, B., Brånemark, P.-I. (1981) A 15-year study of osseointegrated implants in the treatment of the edentulous jaw. International Journal of Oral Surgery 10: 387-416

Ainamo, J. \& Bay, I. (1975) Problems and proposals for recording gingivitis and plaque. International Dental Journal 25: 229-235

Albrektsson, T., Brånemark, P.-I., Hansson, H.A., Lindström, J. (1981)

Osseointegrated titanium implants. Requirements for ensuring a longlasting direct bone anchorage in man. Acta Orthopaedica Scandinavica 52: 155-170

Becker, W., Becker, B., Handelsman, M., Ochsenbein, C., Albrektsson, T. (1991) Guided tissue regeneration for implants placed into extraction sockets: a study in dogs. Journal of Periodontology 62: 703-709

Berglundh, T., Persson, L., Klinge, B. (2002) A systematic review of the incidence of biological and technical complications in implant dentistry reported in prospective longitudinal studies of at least 5 years. Journal of Clinical Periodontology 29 (Suppl 3): $197-212$

Brägger, U., Hämmerle, C.H.F., Lang, N.P. (1997) Immediate transmucosal implants using the principle of guided tissue regeneration. II. A cross-sectional study comparing the clinical outcome 1 year after immediate to standard implant placement. Clinical Oral Implants Research 7: 268-276

Burchardt, H. (1983) The biology of bone graft repair. Clinical Orthopaedics and Related Research 174: 28-42 
Buser, D., Brägger, U., Lang, N.P., Nyman, S. (1990) Regeneration and enlargement of jaw bone using guided tissue regeneration. Clinical Oral Implants Research 1: 2232

Buser, D., Dula, K., Lang, N.P., Nyman, S. (1996) Long-term stability of osseointegrated implants in bone regenerated with the membrane technique. 5-year result of a prospective study with 12 implants. Clinical Oral Implants Research 7: $175-183$

Buser, D., Weber, H.P., Brägger, U., Balsiger, C. (1991) Tissue integration of onestage ITI implants. 3-year results of a longitudinal study with hollow-cylinder and hollow-screw implants. International Journal of Oral and Maxillofacial Implants 6: 405-412

Corrente, G., Abundo, R., Cardaropoli, D., Cardaropoli, G., Martuscelli, G. (2000) Long-term evaluation of osseointegrated implants in regenerated and nonregenerated bone. International Journal of Periodontics \& Restorative Dentistry 20: $391-397$

Dahlin, C., Andersson, L., Linde, A. (1991a) Bone augmentation at fenestrated implants by an osteopromotive membrane technique. A controlled clinical study. Clinical Oral Implants Research 2: 159-165

Dahlin, C., Lekholm, U., Becker, W., Becker, B., Higuchi, K., Callens, A., van Steenberghe, D. (1995) Treatment of fenestration and dehiscence bone defects around oral implants using the guided tissue regeneration technique: a prospective multicenter study. International Journal of Oral and Maxillofacial Implants 10: $312-318$

Dahlin, C., Linde, A., Gottlow, J., Nyman, S. (1988) Healing of bone defects by guided tissue regeneration. Plastic and Reconstructive Surgery 81: 672-677 
Dahlin, C., Sennerby, L., Lekholm, U., Linde, A., Nyman, S. (1989) Generation of new bone around titanium implants using a membrane technique: An experimental study in rabbits. International Journal of Oral and Maxillofacial Implants 4: 19-25

Fiorellini, J.P., Nevins, M.L. (2003) Localized Ridge Augmentation/Preservation. A systematic review. Annals of Periodontology 8: 321-327

Hämmerle, C.H.F., Brägger, U., Schmid, B., Lang, N.P. Successful bone formation at immediate transmucosal implants. International Journal of Oral and Maxillofacial Implants 13: 522-530

Hämmerle, C.H.F., Chen, S.T., Wilson, T.G. (2004) Consensus statements and recommended clinical procedures regarding the placement of implants in extraction sockets. International Journal of Oral and Maxillofacial Implants 19 (Suppl): 26-28

Hämmerle, C.H.F., Feloutzis, A. (2002) A systematic review of the survival of implants in bone sites augmented with barrier membranes (guided bone regeneration) in partially edentulous patients. Journal of Clinical Periodontology 29: $1-6$

Hämmerle, C.H.F., Jung, R.E., Yaman, D., Lang, N.P. (2007) Ridge augmentation by applying bioresorbable membranes and deproteinized bovine bone material. A report of 12 consecutive cases. Clinical Oral Implants Research 19: 19-25

Hämmerle, C.H.F., Olah, A.J., Schmid, J., Flückiger, L., Winkler, J.R., Gogolowski, S., Lang, N.P. (1997) The biological effect of deproteinized bovine bone on bone neoformation on the rabbit skull. Clinical Oral Implants Research 8: 198-207

Hämmerle, C.H.F., Schmid, J., Olah, A.J., Lang, N.P. (1996) A novel model system for the study of experimental bone formation in humans. Clinical Oral Implants Research 7: 38-47

Ilizarov, G.A. (1989a) The tension-stress effect on the genesis and growth of tissues: Part I. The influence of stability of fixation and soft tissue preservation. Clinical Orthopaedics 238: 249-281 
Ilizarov, G.A. (1989b) The tension-stress effect on the genesis and growth of tissues:

Part II. The influence of the rate and frequency of distraction. Clinical Orthopaedics 239: $263-285$

Jemt, T., Lekholm, U. (1993) Oral implant in posterior partially edentulous jaws: a 5year follow-up report. International Journal of Oral and Maxillofacial Implants 8: $635-640$

Kostopoulos, L. \& Karring, T. (1994) Augmentation of the rat mandible using guided tissue regeneration. Clinical Oral Implants Research 5: 75-82

Lang, N.P., Hämmerle, C.H.F., Brägger, U., Lehmann, B., Nyman, S.R. (1994) Guided tissue regeneration in jawbone defects prior to implants placement. Clinical Oral Implants Research 5: 92-97

Lekholm, U., Adell, R., Lindhe, J. (1986) Marginal tissue reactions at osseointegrated titanium fixtures. A cross-sectional retrospective study. International Journal of Oral \& Maxillofacial Surgery 15: 53-61

Lekholm, U., van Steenberghe, D., Hermann, I., Bolender, C., Folmer, T., Gunne, J., Henry, P., Higuchi, K., Laney, W.R., Lindén, U. (1994) Osseointegrated implants in the treatment of partially edentulous jaws: a prospective 5-year multicenter study. International Journal of Oral and Maxillofacial Implants 9: 627-635

Mayfield, L., Skoglund, A., Nobreus, N., Attström, R. (1998) Clinical and radiographic evaluation, following delivery of fixed reconstructions, at GBR treated titanium fixtures. Clinical Oral Implants Research 9: 292-302

Nyman, S.R. \& Lang, N.P. (1994) Guided tissue regeneration and dental implants. Periodontology 2000 4:109-118

Nyman, S., Lang, N.P., Buser, D., Brägger, U. (1990) Bone regeneration adjacent to titanium dental implants using guided tissue regeneration. A report of 2 cases. International Journal of Oral and Maxillofacial Implants 5: 9-14 
O'Leary, T., Drake, R., Naylor, J. (1972) The plaque control record. Journal of Periodontology 43: 38

Pylant, T., Triplett, R.G., Key, M.C., Brunsvold, M.A. (1992) A retrospective evaluation of endosseus titanium implants in the partially edentulous patient. International Journal of Oral and Maxillofacial Implants 7: 195-202

Ramfjord, S.P. (1959) Indices for prevalence and incidence of periodontal deisease. Journal of Periodontology 30: 51-59

Reddi, A.H., Weintroub, S., Muthukumaran, N. (1987) Biologic principles of bone induction. The Orthopedic Clinics of North America 18: 207-212

Rodoni, L.R., Glauser, R., Feloutzis, A., Hämmerle, C.H.F. (2005) Implants in the posterior maxilla: a comparative clinical and radiologic study. International Journal of Oral and Maxillofacia Implants 20: 231-237

Simion, M., Jovanovic, S.A., Tinti, C., Benfenati, S.P. (2001) Long-term evaluation of osseointegrated implants inserted at the time or after vertical ridge augmentation. A retrospective study on 123 implants with 1-5 years follow-up. Clinical Oral Implants Research 12: 35-45

Updegrave, W.J. (1968) Right-angle dental radiography. Dental Clinics of North America 76: 571-579

Urist, M.R. (1965) Bone: Formation by autoinduction. Science 150: 893-899

Weber, H.P., Buser, D., Fiorellini, J.P., Williams, R.C. (1992) Radiographic evaluation of crestal bone levels adjacent to non-submerged titanium implants. Clinical Oral Implants Research 3: 181-188

Weber, H.P., Crohin, C.C., Fiorellini, J.P. (2000) A 5-year prospective clinical and radiolographic study of non-submerged dental implants. Clinical Oral Implants Research 11: 144-153 
Zitzmann, N.U., Schärer, P., Marinello, C.P. (2001) Long-term results of implants treated with guided bone regeneration: a 5-year prospective study. International Journal of Oral \& Maxillofacial Surgery 16: 355-366 